\title{
A systematic review of contemporary evidence on SARS-CoV-2 and HIV coinfection: What does it look like up to date?
}

\author{
Mohammad Abrar Shareef, Hafss M. Bashaiwth, Abdullah O. AlAkbari, Marwan S. Bahamran, Maryam O. AlAmodi, \\ Salem H. Albaiti, Meryam A. Ali, Abdulaziz M. Eshaq, Khaled Alkattan, Abdulhadi A. Alamodi \\ Department of Family Medicine, Sebasticook Valley Hospital, Pittsfield, Maine, USA, ${ }^{1}$ College of Medicine, Alfaisal University, Riyadh, Saudi \\ Arabia, ${ }^{2}$ College of Medicine, Hadhramout University, Mukalla, Yemen, ${ }^{3}$ Jackson State University, ${ }^{4}$ School of Public Health, Jackson State \\ University, Jackson, MS, USA
}

\begin{tabular}{|c|}
\hline Access this article online \\
\hline Website: www.avicennajmed.com \\
\hline DOI: 10.4103/ajm.ajm_175_20 \\
\hline Quick Response Code: \\
\hline
\end{tabular}

\begin{abstract}
Background: Preexisting alteration of the immune system by factors including older age, cardiovascular diseases, morbid obesity, diabetes, and chronic obstructive pulmonary disease (COPD) have detrimental effects on SARS-CoV-2 patients. Literature regarding SARS-CoV-2/ human immunodeficiency virus (HIV) is still developing. Materials and Methods: We reviewed the existing literature pertaining to SARS-CoV-2/HIV coinfection systematically. Research records' characteristics and patients' clinical data were collected. Results: Seven research records were included, of which three were case series and four were case reports, reporting a total of 16 cases. There was one case of death, whereas (15/16) patients were discharged home. Majority of patients developed consistent clinical presentation of SARS-CoV-2. All patients had initial positive RT-PCR results, and four cases had HIV-related lymphopenia. Conclusion: Although the current literature is still growing to increase our understanding of SARS-CoV-2/ HIV coinfection, people living with HIV should adhere to the guidelines of healthy behavior and practice during this pandemic.
\end{abstract}

Key words: Coinfection, COVID-19, HIV, review, SARS-CoV-2

\section{INTRODUCTION}

The severe acute respiratory syndrome coronavirus-2 (SARS$\mathrm{CoV}-2$ ) belongs to a family of pathogens that cause various respiratory illnesses. They are coined as coronaviruses due to the characteristic spike-like proteins on their surface. ${ }^{[1]}$ coronaviruses were first recognized in humans in 1960, and since then seven distinct pathogens were identified. There are four common human coronaviruses: 229E (alpha coronavirus), NL63 (alpha coronavirus), OC43 (beta coronavirus), and HKU1 (beta coronavirus)..$^{[2]}$ Over the past two decades, there were two outbreaks of coronaviruses, namely, the severe acute respiratory syndrome corona virus (SARS-CoV) in 2002-2003, and the Middle East respiratory

Address for correspondence: Dr. Abdulhadi A. AlAmodi, School of Public Health, College of Health Sciences, Department of Epidemiology and Biostatistics, Jackson State University, Jackson, MI 39213, USA.

E-mail: alamoudi.aa89@gmail.com syndrome (MERS) in 2011. ${ }^{[3,4]}$ Relative to coronavirus disease-2019 (COVID-19), SARS and MERS were largely controlled and not catastrophic. The current pandemic of SARS-Cov- 2 has resulted in various deleterious consequences on the public health, economy, and healthcare systems. ${ }^{[5]}$

On December 2019, the Health Commission of Hubei in China reported 27 individuals with pneumonia-like symptoms of unknown origin. Seven days later, the

This is an open access journal, and articles are distributed under the terms of the Creative Commons Attribution-NonCommercial-ShareAlike 4.0 License, which allows others to remix, tweak, and build upon the work non-commercially, as long as appropriate credit is given and the new creations are licensed under the identical terms.

For reprints contact: reprints@medknow.com

Cite this article as: Shareef MA, Bashaiwth HM, AlAkbari AO, Bahamran MS, AlAmodi MO, Albaiti SH, et al. A systematic review of contemporary evidence on SARS-CoV-2 and HIV coinfection: What does it look like up to date?. Avicenna J Med 2020;10:189-97. 
Chinese government announced the identification of new coronavirus, and 4 days later, the reported number of cases had risen to 41 confirmed cases with one death. On January 13, 2020, the first case outside China was reported in Thailand. ${ }^{[6]}$ The death toll had increased significantly and on January 30, 2020, the World Health Organization (WHO) announced a global emergency, and on February 11 SARS-CoV-2 was officially identified to cause COVID-19. On March 11, 2020, the WHO declared COVID-19 as a global pandemic. ${ }^{[7]}$ By the end of April of this year, there were over 3 million cases of SARS-Cov-2 with over 218,024 deaths, and 959,212 recovered cases. Based on the closed cases of SARS-Cov-2, the global death rate is variable with the highest prevalence in the USA, Spain, Italy, France, the United Kingdom (UK), Germany, and Turkey. ${ }^{[8]}$

Several investigations have suggested the role of the host immune system as a target and determinant of the SARS-Cov- 2 course and outcome. ${ }^{[9]}$ Preexisting alteration of the immune system by factors including older age, cardiovascular diseases, morbid obesity, diabetes, and chronic obstructive pulmonary disease (COPD) have detrimental effects on SARS-Cov-2 patients. ${ }^{[10]}$ Therefore, it is logical to assume that individuals with other health problems that impact the immune system such as the human immunodeficiency virus (HIV) would be at higher risk of complications and poor prognosis. Approximately 37.9 million people are living with HIV (PLHIV) with the risk of developing chronic comorbid conditions. ${ }^{[1]}$ SARS-Cov-2 and HIV, although not alike, share similar characteristics as they both target T-lymphocytes and result in lymphopenia. ${ }^{[12]}$ Researchers across the globe continue to increase our understanding of SARS-Cov- 2 characteristics, potential therapeutics, and management. In January of this year, there were 317 PubMed-indexed research records, and by April, it has increased to approximately 8000 research records related to SARS-Cov-2. However, during this pandemic evidence related to SARS-CoV-2 and HIV coinfection is lacking. Herein, we review the current literature pertaining to this subject and summarize the findings coupled with suggested recommendations and implications for future directions.

\section{MATERIALS AND METHODS}

\section{Search strategy}

A comprehensive literature search was performed using PubMed, MEDLINE, WHO Registry, and Cochrane Library for clinical studies published between December 1, 2019 and May 1, 2020. The combination of the following terms was used: COVID, COVID-2, COVID-19, SARSCOV-19 orSARS-COV2, and HIV or AIDS. The search was conducted using PRISMA (Preferred Reporting Item for Systematic Reviews and MetaAnalysis). The search results were initially screened by title and abstract of each study. Full-text articles were then examined.

\section{Inclusion and exclusion criteria}

The inclusion criteria entailed cases with confirmed SARS-Cov-2 infection either via Reverse-Transcriptase Polymerase Chain Reaction (RT-PCR) of nasopharyngeal swab or evidence of positive serum immunohistochemical assay result, presence of HIV infection, publication in English language and availability of clinical data and measured outcomes. The exclusion criteria were cases with unconfirmed SARS-Cov-2 infection, non-HIV patients, publication in a different language, or missing or unavailable data. All authors were involved in this process and any conflict was resolved by discussion to reach consensus.

\section{Reports' quality assessment}

The methodology of each study was then evaluated using data quality assessment tool, developed by Murad et al. ${ }^{[13]}$ [Table 1]. This tool consists of eight questions pertaining to four major domains: selection, ascertainment, causality, and reporting. Each study is scored out of " 4 ," where " 1 " or "2" is considered low quality, " 3 " is moderate and " 4 " represents a high quality. As reporting adverse drug events was not relevant to this study, questions 4,5 , and 6 were omitted.

\section{Collected information and data}

The following information was extracted from each article: first author's name, title, and number of reported cases in each study. The main variables obtained from each case included age and gender of the patient, duration of HIV infection, name of antiretroviral therapy (ART), presence

\begin{tabular}{|c|c|}
\hline Domains & Questions \\
\hline Selection & $\begin{array}{l}\text { I. Does the patient(s) represent(s) the whole experience of the investigator (center) or is the selection method } \\
\text { unclear to the extent that other patients with similar presentation may not have been reported? }\end{array}$ \\
\hline \multirow[t]{2}{*}{ Ascertainment } & 2.Was the exposure adequately ascertained? \\
\hline & 3. Was the outcome adequately ascertained? \\
\hline \multirow[t]{4}{*}{ Causality } & 4.Were other alternative causes that may explain the observation ruled out? $*$ \\
\hline & 5. Was there a challenge/rechallenge phenomenon? $*$ \\
\hline & 6. Was there a dose-response effect? $*$ \\
\hline & 7. Was follow-up long enough for outcomes to occur? \\
\hline Reporting & $\begin{array}{l}\text { 8. Is the case(s) described with sufficient details to allow other investigators to replicate the research or to allow } \\
\text { practitioners make inferences related to their own practice? }\end{array}$ \\
\hline
\end{tabular}


of comorbidities, clinical presentation, duration of illness prior to presentation, and presence of hypoxia on initial presentation. Patient's most recent HIV RNA viral load and CD4 count, initial laboratory workup and imaging findings, different treatment modalities that were provided, last follow-up day, patient's status, and disposition plan were also collected from each case presentation.

\section{Data analysis}

The statistical analysis was performed by calculating mean and standard deviation of continuous variables with data being represented as mean $\pm \mathrm{SD}$. Categorical variables are depicted as numbers and percentages (\%). The statistical analysis was processed using IBM Statistical Package for the Social Sciences (SPSS) software program, version 20.

\section{RESULTS}

\section{Search results}

The search strategy of identified terms in PubMed, MEDLINE, WHO Registry, and Cochrane Library yielded 113 articles.
After removing duplicate studies, the retrieved items narrowed to 83 articles. A total of eight studies met the inclusion criteria. Due to unavailable full text of one article, the final number of eligible studies for analysis was seven: three case series and four case reports, reporting a total of 16 cases [Figure 1]. No other observational or interventional clinical study, or systematic review of a similar scope was found.

\section{Reports' quality assessment results}

The methodological quality assessment tool has been previously used for quality evaluation of case series and case reports in a systematic review. ${ }^{[13]}$ In this present review, 10 cases were scored as fair quality, three reports had good quality and three cases were marked as poor quality [Table 2]. In terms of geographic locations of each case, five patients were in Spain, four in Turkey, three in Italy and four patients were from China, two of which reside in Wuhan City.

\section{Baseline and demographic characteristics}

The mean age of the 16 patients was $42.7 \pm 12.8$ years and all of them were male except for one female patient. Patients

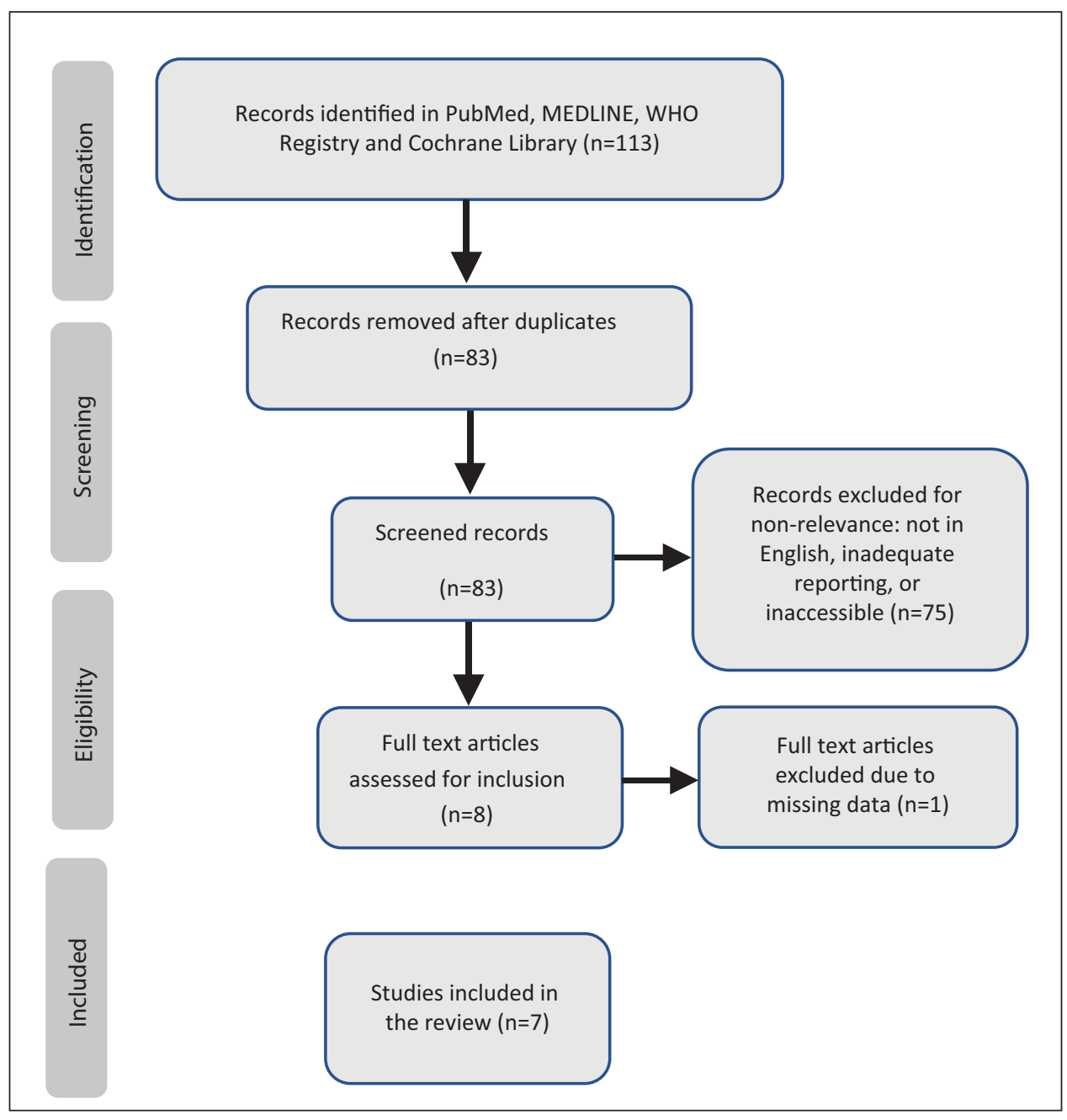

Figure 1: Flow diagram of deployed search strategy 


\begin{tabular}{|c|c|c|c|c|c|c|c|c|c|}
\hline Case & $\begin{array}{c}\text { Author } \\
\text { and } \\
\text { Location }\end{array}$ & $\begin{array}{l}\text { Age } \\
\text {-Gender } \\
\text { (M/F) }\end{array}$ & $\begin{array}{l}\text { Medical } \\
\text { History and } \\
\text { HIV Status }\end{array}$ & $\begin{array}{c}\text { Clinical } \\
\text { Presentation }\end{array}$ & $\begin{array}{l}\text { Imaging } \\
\text { findings }\end{array}$ & Laboratory findings & $\begin{array}{l}\text { Management } \\
\text { Strategy }\end{array}$ & $\begin{array}{l}\text { Outcome } \\
\text { Duration }\end{array}$ & Quality \\
\hline \multirow[t]{2}{*}{1} & $\begin{array}{l}\text { Aydin } \\
\text { et al. } .^{[14]}\end{array}$ & $34 M$ & $\begin{array}{l}\text { HIV X } 10 \text { yrs } \\
\text { HBV Bipolar } \\
\text { disorder }\end{array}$ & $\begin{array}{l}\text { Dyspnea, dry } \\
\text { cough, fever }\end{array}$ & $\begin{array}{l}\text { CT chest: Ground } \\
\text { glass opacities } \\
\text { bilaterally }\end{array}$ & $\begin{array}{c}\text { - HIV RNA: } 434782 \\
\text { copies/mL - CD4: } 2.8 \\
\text { cells/uL - WBC } 2820 \\
\text { cells/uL -Lymphocyte } \\
360 \text { cells/uL - LDH } \\
308 \text { - CRP } 27\end{array}$ & $\begin{array}{c}\text { I) tenofovir/ } \\
\text { emtricitabine+ } \\
\text { lopinavir/ ritonavir }\end{array}$ & $\begin{array}{l}\text { Day 5: } \\
\text { Alive and } \\
\text { discharged } \\
\text { home }\end{array}$ & low \\
\hline & Turkey & & & & & & $\begin{array}{l}\text { 2) azithromycin } \\
\text { 3) TMP-SMX } \\
\text { 4) oseltamivir }\end{array}$ & & \\
\hline \multirow[t]{5}{*}{2} & $\begin{array}{l}\text { Aydin } \\
\text { et al. }{ }^{[14]}\end{array}$ & $44 M$ & $\begin{array}{l}\text { HIV X I } 2 \text { yrs } \\
\text { tenofovir/ } \\
\text { emtricitabine+ } \\
\text { dolutegravir }\end{array}$ & $\begin{array}{l}\text { Dyspnea, dry } \\
\text { cough, fever }\end{array}$ & $\begin{array}{l}\text { CXR and CT } \\
\text { chest: Ground } \\
\text { glass opacities } \\
\text { bilaterally }\end{array}$ & $\begin{array}{l}\text { HIV RNA: } \\
\text { undetectable }\end{array}$ & I) oxygen & $\begin{array}{c}\text { Day 2: } \\
\text { Worsened } \\
\text { and } \\
\text { deceased }\end{array}$ & High \\
\hline & Turkey & & Obese & Hypoxia & & CD4: I 385 cells/uL & $\begin{array}{l}\text { 2) tenofovir/ } \\
\text { emtricitabine+ } \\
\text { dolutegravir }\end{array}$ & & \\
\hline & & & DM & & & $\begin{array}{l}\text { Lymphocyte: } 670 \text { cells/ } \\
\text { uL }\end{array}$ & 3) azithromycin & & \\
\hline & & & COPD & & & LDH 575 & 4) oseltamivir & & \\
\hline & & & HTN & & & $\begin{array}{c}\text { CRP I52 } \\
\text { D-dimer I.5 }\end{array}$ & & & \\
\hline \multirow[t]{3}{*}{3} & $\begin{array}{l}\text { Aydin } \\
\text { et al. } .^{[14]}\end{array}$ & $35 M$ & $\begin{array}{l}\text { HIV tenofovir/ } \\
\text { emtricitabine } \\
\text { +elvitegravir/ } \\
\text { cobicistat }\end{array}$ & $\begin{array}{l}\text { Weakness, dry } \\
\text { cough, watery } \\
\text { diarrhea } x \\
\text { II days }\end{array}$ & $\begin{array}{l}\text { CT chest: Ground } \\
\text { glass opacities } \\
\text { bilaterally }\end{array}$ & $\begin{array}{l}\text { HIV RNA: } \\
\text { undetectable }\end{array}$ & $\begin{array}{c}\text { I) tenofovir/ } \\
\text { emtricitabine } \\
\text { +elvitegravir/ cobicistat }\end{array}$ & $\begin{array}{l}\text { Day 7: } \\
\text { Alive and } \\
\text { improved }\end{array}$ & Moderate \\
\hline & Turkey & & & & & CD4: 448 cells/uL & 2) hydroxychloroquine & $\begin{array}{l}\text { Discharged } \\
\text { home }\end{array}$ & \\
\hline & & & & & & CRP elevated & 3) oseltamivir & & \\
\hline \multirow[t]{3}{*}{4} & $\begin{array}{l}\text { Aydin } \\
\text { et al. }{ }^{[14]}\end{array}$ & $36 \mathrm{M}$ & $\begin{array}{l}\text { HIV tenofovir/ } \\
\text { emtricitabine } \\
\text { +elvitegravir/ } \\
\text { cobicistat }\end{array}$ & $\begin{array}{l}\text { Fever and dry } \\
\text { cough } \times 6 \text { days }\end{array}$ & $\begin{array}{l}\text { CT chest: Ground } \\
\text { glass opacities } \\
\text { bilaterally }\end{array}$ & $\begin{array}{l}\text { HIV RNA: } \\
\text { undetectable }\end{array}$ & $\begin{array}{c}\text { I) tenofovir/ } \\
\text { emtricitabine } \\
\text { +elvitegravir/ cobicistat }\end{array}$ & $\begin{array}{l}\text { Day 7: } \\
\text { Alive and } \\
\text { discharged } \\
\text { home }\end{array}$ & Moderate \\
\hline & Turkey & & & & & CD4 396 cells/uL & 2) azithromycin & & \\
\hline & & & & & & $\begin{array}{c}\text { Lymphocytes } 900 \text { cells/uL } \\
\text { CRP elevated }\end{array}$ & $\begin{array}{l}\text { 3) hydroxychloroquine } \\
\text { 4) oseltamivir }\end{array}$ & & \\
\hline \multirow[t]{3}{*}{5} & $\begin{array}{l}\text { Wang } \\
\text { et al..[15] }\end{array}$ & $37 \mathrm{M}$ & $\begin{array}{l}\text { HIV on ART } \\
\text { Syphilis }\end{array}$ & $\begin{array}{l}\text { Fever, dry } \\
\text { cough and } \\
\text { chest pain } x \\
\text { I month }\end{array}$ & $\begin{array}{l}\text { CT chest: bilateral } \\
\text { infiltrations }\end{array}$ & $\begin{array}{l}\text { RT-PCR COVOD-19 } \\
\text { negative } x 3 \text {, positive } \\
\text { on fourth }\end{array}$ & I) high flow oxygen & $\begin{array}{l}\text { Day 26: } \\
\text { Alive and } \\
\text { inpatient }\end{array}$ & Moderate \\
\hline & $\begin{array}{l}\text { Wuhan, } \\
\text { China }\end{array}$ & & & Hypoxia & & CD4: 34 cells/uL & 2) umifenovir & & \\
\hline & & & & & & $\begin{array}{l}\text { Lymphocyte: normal } \\
\text { CRP elevated }\end{array}$ & $\begin{array}{l}\text { 3) methylprednisolone } \\
\text { 4) moxifloxacin } \\
\text { 5) sulbactam/ } \\
\text { cefoperazone } \\
\text { 6) tocilizumab }\end{array}$ & & \\
\hline \multirow[t]{4}{*}{6} & $\begin{array}{l}\text { Riva } \\
\text { et al. }{ }^{[16]}\end{array}$ & $62 M$ & $\begin{array}{l}\text { HIV } \\
\text { darunavir/ } \\
\text { cobicistat }+ \\
\text { lamivudine }\end{array}$ & $\begin{array}{l}\text { Dry cough, } \\
\text { fever } \times \text { I wk }\end{array}$ & $\begin{array}{l}\text { CXR: bilateral } \\
\text { infiltrations }\end{array}$ & $\begin{array}{c}\text { HIV RNA: }<20 \text { copies/ } \\
\text { mL }\end{array}$ & $\begin{array}{l}\text { I) oxygen -> CPAP -> } \\
\text { mechanical ventilation }\end{array}$ & $\begin{array}{l}\text { Day 20: } \\
\text { Alive and } \\
\text { inpatient }\end{array}$ & Moderate \\
\hline & Italy & & HTN & & & CD4: 44I cells/uL & 2) lopinavir/ritonavir & & \\
\hline & & & CAD & & & $\begin{array}{l}\text { Darunavir: therapeutic } \\
\text { range }\end{array}$ & 2) tocilizumab & & \\
\hline & & & & & & & $\begin{array}{l}\text { 3) remdesivir } \\
\text { 4) hydroxychloroquine }\end{array}$ & & \\
\hline \multirow[t]{3}{*}{7} & $\begin{array}{l}\text { Riva } \\
\text { et al. } .^{[16]}\end{array}$ & $63 M$ & HIV & Fever $\times$ II days & $\begin{array}{l}\text { CXR: bilateral } \\
\text { reticular } \\
\text { interstitial } \\
\text { thickening }\end{array}$ & $\begin{array}{c}\text { HIV RNA: }<20 \text { copies/ } \\
\text { mL }\end{array}$ & $\begin{array}{l}\text { 1) lopinavir/ } \\
\text { ritonavir + tenofovir/ } \\
\text { emtricitabine }\end{array}$ & $\begin{array}{l}\text { Day 10: } \\
\text { Alive and } \\
\text { discharged } \\
\text { home }\end{array}$ & Low \\
\hline & Italy & & $\begin{array}{l}\text { Darunavir/ } \\
\text { cobicistat, } \\
\text { tenofovir + } \\
\text { emtricitabine }\end{array}$ & $\begin{array}{l}\text { Positive history } \\
\text { of contact with } \\
\text { COVID patient }\end{array}$ & & CD4: 743 cells/uL & 2) hydroxychloroquine & & \\
\hline & & & HTN & & & $\begin{array}{l}\text { Darunavir: therapeutic } \\
\text { range }\end{array}$ & & & \\
\hline
\end{tabular}




\begin{tabular}{|c|c|c|c|c|c|c|c|c|c|}
\hline Case & $\begin{array}{l}\text { Author } \\
\text { and } \\
\text { Location }\end{array}$ & $\begin{array}{l}\text { Age } \\
\text {-Gender } \\
\text { (M/F) }\end{array}$ & $\begin{array}{l}\text { Medical } \\
\text { History and } \\
\text { HIV Status }\end{array}$ & $\begin{array}{c}\text { Clinical } \\
\text { Presentation }\end{array}$ & $\begin{array}{l}\text { Imaging } \\
\text { findings }\end{array}$ & Laboratory findings & $\begin{array}{l}\text { Management } \\
\text { Strategy }\end{array}$ & $\begin{array}{l}\text { Outcome } \\
\text { Duration }\end{array}$ & Quality \\
\hline \multirow[t]{2}{*}{8} & $\begin{array}{l}\text { Riva } \\
\text { et al. } .^{[16]}\end{array}$ & $57 \mathrm{~F}$ & $\begin{array}{l}\text { HIV darunavir/ } \\
\text { cobicistat }+ \\
\text { raltegravir }\end{array}$ & $\begin{array}{l}\text { Fever and } \\
\text { cough } \times 10 \text { days }\end{array}$ & $\begin{array}{c}\text { CXR: reticular } \\
\text { interstitial } \\
\text { thickening at right } \\
\text { lung }\end{array}$ & $\begin{array}{l}\text { Darunavir: therapeutic } \\
\text { range }\end{array}$ & $\begin{array}{l}\text { I) darunavir/ cobicistat } \\
+ \text { raltegravir }\end{array}$ & $\begin{array}{c}\text { Day 7: } \\
\text { Alive and } \\
\text { inpatient }\end{array}$ & Low \\
\hline & Italy & & HTN & & & & 2) hydroxychloroquine & & \\
\hline \multirow[t]{2}{*}{9} & $\begin{array}{l}\text { Blanco } \\
\text { et al. } .^{[1]]}\end{array}$ & $40 M$ & $\begin{array}{l}\text { HIV } \times \text { I3 yrs } \\
\text { tenofovir/ } \\
\text { emtricitabine } \\
\text { + darunavir/ } \\
\text { cobicistat }\end{array}$ & $\begin{array}{l}\text { Fever, cough, } \\
\text { malaise and } \\
\text { headache } x \\
2 \text { days }\end{array}$ & CXR: normal & $\begin{array}{c}\text { HIV RNA: }<50 \text { copies } / \\
\mathrm{mL}\end{array}$ & $\begin{array}{c}\text { I) tenofovir, } \\
\text { emtricitabine }+ \\
\text { darunavir/ cobicistat }\end{array}$ & $\begin{array}{l}\text { Day I: } \\
\text { alive and } \\
\text { treated }\end{array}$ & Moderate \\
\hline & Spain & & & & & $\begin{array}{l}\text { CD4: } 616 \text { cells/uL } \\
\text { WBC: } 7840 \text { cells/uL } \\
\text { Lymphocyte: } 2700 \\
\text { cells/uL }\end{array}$ & & & \\
\hline \multirow[t]{5}{*}{10} & $\begin{array}{l}\text { Blanco } \\
\text { et al. } .^{[17]}\end{array}$ & $49 M$ & HIV x 17 yrs & $\begin{array}{c}\text { Fever and } \\
\text { cough } \times 5 \text { days }\end{array}$ & $\begin{array}{l}\text { CXR: Ground } \\
\text { glass opacities } \\
\text { bilaterally }\end{array}$ & $\begin{array}{c}\text { HIV RNA: }<50 \text { copies/ } \\
\text { mL }\end{array}$ & $\begin{array}{c}\text { I) Oxygen -> } \\
\text { mechanical ventilation }\end{array}$ & $\begin{array}{l}\text { Day } 21 \text { : } \\
\text { alive, on } \\
\text { ECMO }\end{array}$ & High \\
\hline & Spain & & $\begin{array}{l}\text { Abacavir/ } \\
\text { lamivudine + } \\
\text { dolutegravir }\end{array}$ & Hypoxia & & CD4: 445 cells/uL & $\begin{array}{c}\text { 2) tenofovir / } \\
\text { emtricitabine }+ \\
\text { lopinavir/ritonavir }\end{array}$ & & \\
\hline & & & & & & WBC: 29160 cells/uL & $\begin{array}{l}\text { 3) meropenem/ } \\
\text { linezolid }\end{array}$ & & \\
\hline & & & & & & $\begin{array}{l}\text { Lymphocyte: I I } 70 \\
\text { cells/uL }\end{array}$ & 4) hydroxychloroquine & & \\
\hline & & & & & & LDH 316 & $\begin{array}{l}\text { 5) interferon beta- } \\
\text { Ib }\end{array}$ & & \\
\hline \multirow[t]{3}{*}{11} & $\begin{array}{l}\text { Blanco } \\
\text { et al.[17] }\end{array}$ & $29 M$ & HIV $\times 7$ yrs & $\begin{array}{l}\text { Fever, malaise, } \\
\text { cough, headache, } \\
\text { dyspnea } x 2 \text { days }\end{array}$ & CXR: normal & $\begin{array}{c}\text { CRP } 30 \\
\text { HIV RNA: }<50 \text { copies/ } \\
\mathrm{mL}\end{array}$ & $\begin{array}{l}\text { 6) tocilizumab } \\
\text { I) tenofovir/ } \\
\text { emtricitabine + } \\
\text { lopinavir/ ritonavir }\end{array}$ & $\begin{array}{l}\text { Day 3: } \\
\text { Alive and } \\
\text { resolved }\end{array}$ & Moderate \\
\hline & Spain & & $\begin{array}{c}\text { Tenofovir/ } \\
\text { emtricitabine } \\
\text { + darunavir/ } \\
\text { cobicistat }\end{array}$ & & & CD4: 604 cells/uL & 2) hydroxychloroquine & & \\
\hline & & & & & & $\begin{array}{l}\text { WBC: } 6730 \text { cells/uL } \\
\text { Lymphocyte: I } 500 \\
\text { cells/uL } \\
\text { LDH } 256 \\
\text { CRP } 72\end{array}$ & 3) azithromycin & & \\
\hline \multirow[t]{3}{*}{12} & $\begin{array}{l}\text { Blanco } \\
\text { et al. } .^{[1]]}\end{array}$ & $40 M$ & HIV x 17 yrs & $\begin{array}{l}\text { Fever, malaise, } \\
\text { cough, } \\
\text { headache, } \\
\text { dyspnea } \\
\text { x3 days }\end{array}$ & $\begin{array}{l}\text { CXR: right } \\
\text { basal interstitial } \\
\text { infiltrate }\end{array}$ & $\begin{array}{c}\text { HIV RNA: }<50 \text { copies } / \\
\mathrm{mL}\end{array}$ & $\begin{array}{c}\text { I) tenofovir/ } \\
\text { emtricitabine + } \\
\text { lopinavir/ ritonavir }\end{array}$ & $\begin{array}{l}\text { Day 4: } \\
\text { Alive and } \\
\text { resolved }\end{array}$ & Moderate \\
\hline & Spain & & $\begin{array}{l}\text { Abacavir, } \\
\text { lamivudine; } \\
\text { and } \\
\text { dolutegravir }\end{array}$ & & & CD4: II40 cells/uL & 2) hydroxychloroquine & & \\
\hline & & & & & & $\begin{array}{l}\text { WBC: } 6 \text { I } 40 \text { cells/uL } \\
\text { Lymphocyte: I } 4670 \\
\text { cells/uL } \\
\text { LDH } 465 \\
\text { CRP } 0.43\end{array}$ & $\begin{array}{l}\text { 3) azithromycin } \\
\text { 4) cefixime }\end{array}$ & & \\
\hline 13 & $\begin{array}{l}\text { Blanco } \\
\text { et al. }{ }^{[1]} \\
\text { Spain }\end{array}$ & $31 \mathrm{M}$ & $\begin{array}{c}\text { HIV } \\
\text { No ART }\end{array}$ & $\begin{array}{l}\text { Fever, cough, } \\
\text { dyspnea } \\
\text { x7 days } \\
\text { Hypoxia }\end{array}$ & $\begin{array}{l}\text { CXR: right basal } \\
\text { pneumonia with } \\
\text { pleural effusion }\end{array}$ & $\begin{array}{c}\text { HIV RNA: } 4550 \\
\text { copies/ } \\
\mathrm{mL} \\
\text { CD4: I } 3 \text { cells/uL } \\
\text { WBC: I } 4670 \text { cells/uL } \\
\text { Lymphocyte: } 900 \text { cells/ } \\
\text { uL } \\
\text { LDH I I } 49 \\
\text { CRP } 40\end{array}$ & $\begin{array}{l}\text { I) tenofovir/ } \\
\text { emtricitabine }+ \\
\text { darunavir/ cobicistat } \\
\text { 2) interferon beta- } \\
\text { Ib } \\
\text { 3) hydroxychloroquine } \\
\text { 4) azithromycin } \\
\text { 5) ceftaroline } \\
\text { 6) co-trimoxazole } \\
\text { 7) corticosteroids }\end{array}$ & $\begin{array}{l}\text { Day } 12 \text { : } \\
\text { alive and } \\
\text { resolved }\end{array}$ & Moderate \\
\hline
\end{tabular}




\begin{tabular}{|c|c|c|c|c|c|c|c|c|c|}
\hline Case & $\begin{array}{l}\text { Author } \\
\text { and } \\
\text { Location }\end{array}$ & $\begin{array}{c}\text { Age } \\
\text {-Gender } \\
\text { (M/F) }\end{array}$ & $\begin{array}{l}\text { Medical } \\
\text { History and } \\
\text { HIV Status }\end{array}$ & $\begin{array}{c}\text { Clinical } \\
\text { Presentation }\end{array}$ & $\begin{array}{l}\text { Imaging } \\
\text { findings }\end{array}$ & Laboratory findings & $\begin{array}{l}\text { Management } \\
\text { Strategy }\end{array}$ & $\begin{array}{l}\text { Outcome } \\
\text { Duration }\end{array}$ & Quality \\
\hline \multirow[t]{2}{*}{14} & $\begin{array}{l}\text { Chen } \\
\text { et al. } .^{[18]}\end{array}$ & $24 M$ & $\begin{array}{l}\text { HIV } \times 2 \text { yrs } \\
\text { tenofovir/ } \\
\text { lamivudine } \\
\text { +efavirenz }\end{array}$ & $\begin{array}{c}\text { Fever, dry } \\
\text { cough } \times \text { I day }\end{array}$ & $\begin{array}{l}\text { CT chest: multiple } \\
\text { high-density } \\
\text { patchy shadows at } \\
\text { right lung }\end{array}$ & $\begin{array}{l}\text { RT-PCR COVID-19: } \\
\text { positive on second } \\
\text { test }\end{array}$ & $\begin{array}{c}\text { I) tenofovir/ } \\
\text { lamivudine + efavirenz }\end{array}$ & $\begin{array}{c}\text { Day I5: } \\
\text { alive and } \\
\text { discharged }\end{array}$ & Moderate \\
\hline & China & & & & & $\begin{array}{l}\text { WBC: } \text { normal } \\
\text { Lymphocyte: normal } \\
\text { CRP: normal }\end{array}$ & $\begin{array}{l}\text { 2) lopinavir/ ritonavir } \\
\text { 3) interferon }\end{array}$ & & \\
\hline \multirow[t]{3}{*}{15} & $\begin{array}{l}\text { Zhao } \\
\text { et al. }{ }^{[19]}\end{array}$ & $38 M$ & $\begin{array}{l}\text { HIV tenofovir/ } \\
\text { lamivudine } \\
\text { +efavirenz }\end{array}$ & Fever, malaise & $\begin{array}{l}\text { CT chest: right } \\
\text { lower lobe } \\
\text { pneumonia }\end{array}$ & $\begin{array}{c}\text { RT-PCR SARS COV-I9: } \\
\text { negative } \times 3\end{array}$ & $\begin{array}{c}\text { I) tenofovir/ } \\
\text { lamivudine +efavirenz }\end{array}$ & $\begin{array}{l}\text { Day 45: } \\
\text { Alive and } \\
\text { improved }\end{array}$ & High \\
\hline & China & & $\mathrm{HCV}$ & $\begin{array}{l}\text { Positive travel } \\
\text { history to } \\
\text { Wuhan, China }\end{array}$ & & $\begin{array}{l}\text { IgM positive after } \\
42 \text { days with negative } \\
\text { repeat RT-PCR }\end{array}$ & 2) oseltamivir & & \\
\hline & & & & & & $\begin{array}{c}\text { HIV RNA: }<500 \\
\text { copies/ } \\
\text { mL } \\
\text { HCV RNA: }<500 \\
\text { copies } / \mathrm{mL} \\
\text { CD4: } 84 \text { cells/uL } \\
\text { WBC: } 2800 \text { cells/uL } \\
\text { CRP } 16.6 \mathrm{mg} / \mathrm{L}\end{array}$ & 3) interferon & & \\
\hline \multirow[t]{3}{*}{16} & $\begin{array}{l}\text { Zhu } \\
\text { et al. }{ }^{[20]}\end{array}$ & $61 M$ & HIV & $\begin{array}{l}\text { Fever, dry } \\
\text { cough and } \\
\text { dyspnea } x \\
6 \text { days }\end{array}$ & $\begin{array}{l}\text { CT chest: ground } \\
\text { glass opacities } \\
\text { bilaterally }\end{array}$ & $\begin{array}{c}\text { Lymphocyte: I I } 00 \\
\text { cells/uL }\end{array}$ & I) oxygen & $\begin{array}{l}\text { Day 2I: } \\
\text { alive and } \\
\text { discharged } \\
\text { home }\end{array}$ & Moderate \\
\hline & $\begin{array}{l}\text { Wuhan, } \\
\text { China }\end{array}$ & & DM & Hypoxia & & & 2) lopinavir/ ritonavir & & \\
\hline & & & Smoker & & & & $\begin{array}{l}\text { 3) moxifloxacin } \\
\text { 4) } \gamma \text {-globulin } \\
\text { 5) methylprednisolone }\end{array}$ & & \\
\hline
\end{tabular}

$\mathrm{CAD}=$ coronary artery disease, $\mathrm{COPD}=$ chronic obstructive pulmonary disease, COVID-19 = coronavirus disease $2019, \mathrm{CPAP}=$ continuous positive airway pressure, $\mathrm{CRP}=\mathrm{C}$-reactive protein, $\mathrm{CT}=$ computed tomography, $\mathrm{CXR}=$ chest $\mathrm{X}$-ray, $\mathrm{DM}=$ diabetes mellitus, $\mathrm{ECMO}=$ extracorporeal membrane oxygenation, $\mathrm{HBV}=$ hepatitis $\mathrm{C}$ virus, $\mathrm{HCV}=$ hepatitis $\mathrm{C}$ virus, $\mathrm{HIV}=$ human-immunodeficiency virus, $\mathrm{HTN}=$ hypertension, $\mathrm{IgM}=$ immunoglobulin $\mathrm{M}, \mathrm{LDH}=$ lactate dehydrogenase, $\mathrm{mL}=$ milliliter, $\mathrm{RNA}=$ ribonucleic acid, $\mathrm{RT}-\mathrm{PCR}=$ real-time polymerase chain reaction, $\mathrm{TMP}-\mathrm{SMX}=$ trimethoprim/sulfamethoxazole, $\mu \mathrm{L}=$ microliter, $\mathrm{WBC}=$ white blood cells

had HIV disease for an average of $10.2 \pm 5.2$ years and 13 out of 16 patients were taking ART at home with documented compliance and suppressed viral load. One patient had poor compliance to treatment due to underlying bipolar disorder and two patients were not on ART prior to presentation with COVID-19 symptoms. About eight cases had a reduced CD4 level of less than 500 cells/microliter. With regards to comorbid conditions, four patients had a history of hypertension, two patients with diabetes mellitus and one obese patient. One patient had a history of syphilis, whereas another case reported a history of hepatitis $\mathrm{C}$ virus (HCV) coinfection [Table 2].

\section{Clinical presentation}

Most of the patients presented with fever (94\%) followed by dry cough (88\%) and dyspnea (34\%) as shown in Figure 2. The mean duration of illness on presentation was $10.17 \pm 5.19$ days. A total of five patients had hypoxia and required oxygen per nasal cannula. Two patients required mechanical ventilation: one has improved, whereas the second one was eventually placed on extracorporeal membrane oxygenation (ECMO) [Table 2].

\section{Screening and laboratory findings}

A vast majority of patients had a positive RT-PCR result on initial testing (81\%), whereas the remaining patients had initially persisted negative RT-PCR results. Upon evaluation of initial laboratory findings, two patients had leukocytosis on initial workup, two others had leukopenia and four cases reported lymphopenia. Despite only five studies reporting lactate dehydrogenase level (LDH), all patients had LDH greater than 200 units/L. Furthermore, half of the cases had elevated C-reactive protein in their initial laboratory workup [Table 2].

\section{Computed tomography imaging results}

The most common imaging finding in the enrolled cases was bilateral ground-glass opacities in either chest X-ray or CT chest results $(n=8)$. Five patients showed a unilateral infiltrate and two others had a normal imaging result. CT chest of one patient showed multiple high-density patchy shadows at the right lung that resolved following treatment. 


\section{Outcome and selected therapeutics}

Five patients underwent change of ART regimen, whereas one patient with recent HIV diagnosis was started on ART after the SARS-Cov-2 diagnosis. Twelve patients were on tenofovir and emtricitabine combination in addition to other medication combinations. About half of the cases were taking lopinavir and ritonavir combination. Hydroxychloroquine was frequently administered among SARS-Cov-2 infected HIV patients (56\%) with QTc monitoring. The most common antibiotic that was used for COVID-19 therapy or prophylaxis for opportunistic infection was azithromycin (44\%) followed by sulfonamide (13\%). Other antibiotics that were administered include moxifloxacin, meropenem, linezolid, sulbactam/ cefoperazone, cefixime, and caftarolene. Furthermore, around $38 \%$ of patients required immunosuppressive medications either steroid or tocilizumab [Table 2]. The average time interval between disease onset and eradication of this virus, via procuring a negative RT-PCR testing result after showing clinical improvement, was around 14 days. For instance, seven cases documented resolution in less than or equal to 1 -week period, whereas one patient had a slower recovery with interval of 45 days [Table 2]. Overall, all the patients have improved with the provided treatment regimens except for one patient with comorbid conditions.

\section{DISCUSSION}

The current review provides a preliminary evidence that HIV patients coinfected with SARS-Cov-2 developed clinical symptoms consistent with non-HIV individuals with SARS-Cov-2 (fever, dry cough, and dyspnea) [Figure 2]. . ${ }^{[21]}$ The average duration between clinical symptoms onset and resolution was 14 days. Fifteen of 16 patients had resolved SARS-Cov-2 following treatment where they were discharged to home or transferred to inpatient [Table 2 ]. The mortality and fatality of SARS-Cov- 2 is close to $2 \%$; however, it is known that older individuals with comorbid conditions have a poor prognosis toward the disease. ${ }^{[10]}$ In this review, the only death outcome was reported for a 44-year-old male from Turkey, with normal CD4 count and suppressed viral load, who had a history of obesity, diabetes mellitus, COPD, and hypertension. These findings are consistent with reports published in literature regarding COVID-19 clinical presentation and illness duration in non-HIV patients. ${ }^{[21]}$ It is recommended that individuals with chronic comorbid conditions adhere to healthy behavior and practice that leads to controlling such chronic conditions. ${ }^{[10]}$

Although findings should be interpreted with caution, it has been suggested that favorable outcome, or not as worse as thought, of SARS-Cov-2 /HIV coinfection could be attributed to role of ART. In this compiled literature, most patients were on lopinavir and ritonavir combination and tenofovir and emtricitabine combination. Lim et al. ${ }^{[22]}$ proposed that lopinavir-boosted ritonavir may decrease the viral load and improve the clinical course of SARSCov-2-19. On the contrary, Cao et al. ${ }^{[23]}$ reported one of the early clinical trials on the effectiveness of lopinavirboosted ritonavir, a protease inhibitor, as monotherapy against SARS-Cov-2, where lopinavir-boosted ritonavir did not improve SARS-Cov-2 clinical course or outcome. Darunavir, another protease inhibitor, was also ineffective against SARS-Cov-2 attributed to its low affinity to the protease enzyme. ${ }^{[16]}$ Although the evidence remains largely variable, it is early to judge the effectiveness of applying various HIV-related therapeutics to SARS-Cov-2. The future findings of almost over 15 ongoing clinical trials will elucidate and rectify the current evidence on HIV therapeutics' application to SARS-Cov-2. ${ }^{[24]}$ During this

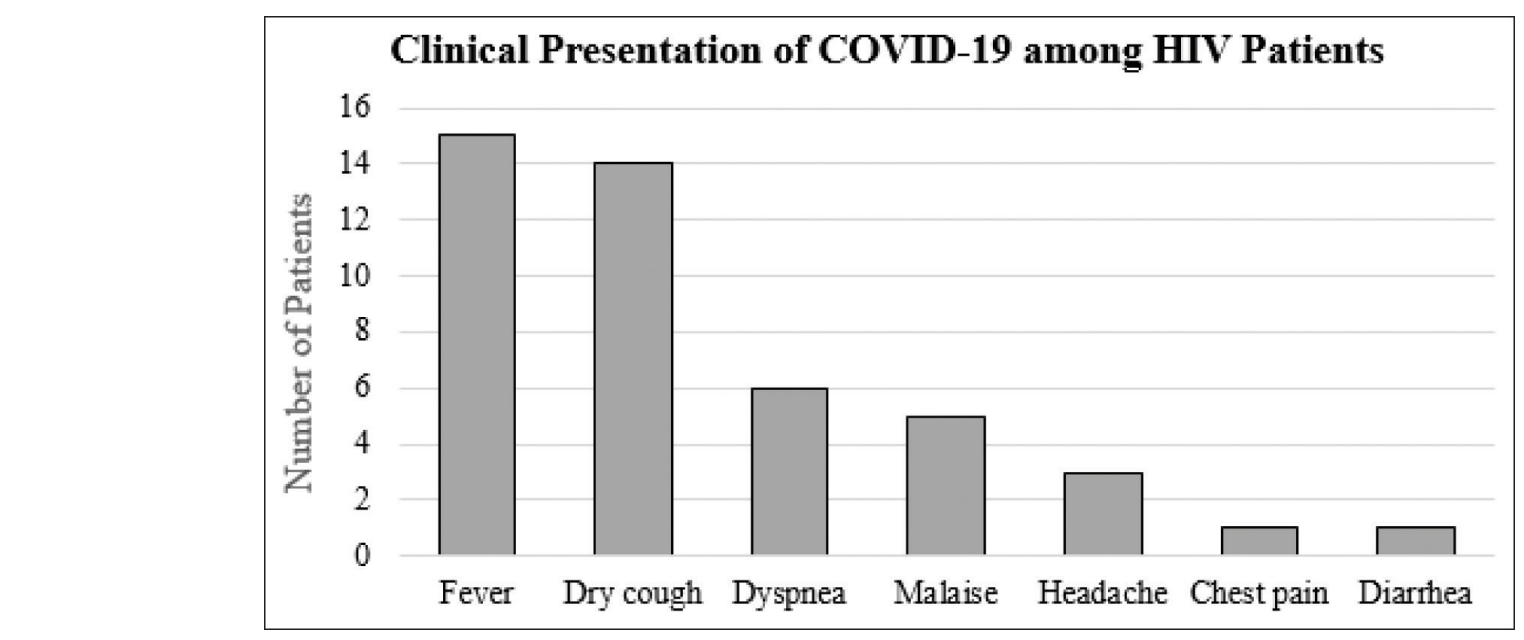

Figure 2: Clinical manifestations of COVID-19 in patients with history of HIV 
period of COVID-19 growing evidence, PLHIV should be compliant and adhere to regularly taking ART and consult with physicians before switching, adding, or removing in the regimen of ART. ${ }^{[24]}$

There seems to be a paradoxical view on the role of lymphopenia in the clinical course and outcome of SARSCov-2. Several studies have shown a consistent pattern of correlation between level of T-lymphocytes and severity of SARS-Cov-2 suggesting that SARS-Cov-2 related lymphopenia carries poor prognosis. ${ }^{[25]}$ This phenomenon has been investigated during the MERS outbreak previously and confirmed a direct invasion to $\mathrm{CD} 4^{+} \mathrm{T}$ and $\mathrm{CD} 8^{+} \mathrm{T}$ cells by MERS-CoV. ${ }^{[25]}$ On the contrary, in this present review there were four cases presented with lymphopenia, including one without ART, that showed good outcomes. Mascolo et al. ${ }^{[26]}$ suggested that the robust activation of the immune system by SARS-Cov-2 is in large part responsible for the tremendous injury to lungs; however, this response may have been curbed by preexisting HIV related lymphopenia. Future research studies are warranted to test this hypothesis and illuminate the paradoxical role of lymphopenia in the course and outcome of SARS-Cov-2 in PLHIV.

The results of RT-PCR testing on collected nasopharyngeal swaps are considered currently the gold start tool to diagnose SARS-Cov-2. All cases presented cases in this review had positive RT-PCR confirming SARS-Cov-2 diagnoses. However, a challenge was presented in two cases where the initial test results of RT-PCR were negative. Although the design of RT-PCR testing was developed to reduce false-negative results, previous reports have documented overall false-negative results due to samples' contamination and primers' mutation. ${ }^{[27]}$ Thus, standards for high standard laboratory practice and technicians' training should be implemented. However, in PLHIV another view was developed for potential false-negative results related to anti-HIV treatment or increased levels of type I interferon (IFN-I). It has been suggested previously that IFN-I, which could be potentially elevated in HIV patients, may contribute to suppressing SARS-CoV-2, and hence below detectable RNA levels. ${ }^{[28]}$ According to a study from China with limited data, SARS-CoV-2 triggers the formation of both IgM, appearing by day 3 , and IgG, appearing by day 8 , in approximately $95 \%$ of COVID-19 patients. ${ }^{[29]} \mathrm{Li}$ et al. ${ }^{[30]}$ have suggested that due to immune response differences, the antibody production may be altered and result in negative blood immunoglobulins. On the contrary, two cases in this review showed persistent detection of antibodies after 42 days and 2 months. Such a delay in antibody clearance and viral elimination explain the long course of disease due to synergistic altered immune system function by HIV and SARS-CoV-2. Therefore, both RT-PCR and antibody laboratory results should be cautiously interpreted by clinical care providers in patients with SARS-Cov-2 /HIV co-infection. Future studies of SARS-Cov-2 in PLHIV are warranted to expand on existing findings.

The typical characteristic of CT chest in COVID-19 patients is ground-glass opacities of both lungs with the peripheral distribution ${ }^{[31]}$ Likewise, in this review, 8 patients presented with ground-glass opacities with bilateral involvement on $\mathrm{CT}$, whereas 5 had a unilateral infiltration and two had a normal CT chest. It is noted that in SARS-Cov- 2 the bilateral lung involvement is more common than unilateral which is more common in MERS and influenza. ${ }^{[31]}$ The absence of any signs may be attributed to the role of ART which has been previously suggested to play a role in improving the absorption capacity of pulmonary changes on CT. ${ }^{[18]} \mathrm{CT}$ chest is an important modality for detecting early changes of SARS-Cov-2 especially in the presence of persistent negative RT-PCR.

\section{CONCLUSION}

Whilst the present findings in this review are preliminary; however, it provides insight into current evidence and opportunities to resolve uncertainty of SARS-Cov-2/HIV co-infection. We summarize and highlight the implications and future directions based on contemporary literature evidence as follows:

- PLHIV co-infected with SARS-Cov-2 should follow the country guidelines of social distancing, self-isolation, and apply consistent hygiene practice.

- PLHIV co-infected with SARS-Cov-2 and have chronic conditions shall practice healthy behavior and practice to control such conditions.

- Future research directions shall aim at the understanding role of lymphopenia, testing pattern and results' consistency, outcome, and HIV-based therapeutics in SARS-Cov-2 /HIV coinfection.

- Although there is no distinct morbidity and mortality, PLHIV shall be on regular ART and shall not change the ART regimen until consulted with physicians.

- Treatment plan for SARS-Cov-2 /HIV patients shall address the psychological burden, and policy should ensure access to healthcare and treatment availability for such a vulnerable group.

\section{Acknowledgement}

The authors would like to thank Prof. Steven Lobello, professor of psychology, at Auburn University at Montgomery 
for providing linguistic suggestions and improving the manuscript.

\section{Financial support and sponsorship}

Nil.

\section{Conflicts of interest}

There are no conflicts of interest.

\section{REFERENCES}

1. Enjuanes L, Almazán F, Sola I, Zuñiga S. Biochemical aspects of coronavirus replication and virus-host interaction. Annu Rev Microbiol 2006;60:211-30.

2. Weiss SR, Navas-Martin S. Coronavirus pathogenesis and the emerging pathogen severe acute respiratory syndrome coronavirus. Microbiol Mol Biol Rev 2005;69:635-64.

3. Ksiazek TG, Erdman D, Goldsmith CS, Zaki SR, Peret T, Emery S, et al.; SARS Working Group. A novel coronavirus associated with severe acute respiratory syndrome. N Engl J Med 2003;348:1953-66.

4. Zaki AM, van Boheemen S, Bestebroer TM, Osterhaus AD, Fouchier RA. Isolation of a novel coronavirus from a man with pneumonia in Saudi Arabia. N Engl J Med 2012;367:1814-20.

5. Nicola M, Alsafi Z, Sohrabi C, Kerwan A, Al-jabir A, losifidis C, et al. The socio-economic implications of the coronavirus and covid-19 pandemic: A review. Int J Surg 2020;78:185-93.

6. Raoofi A, Takian A, Akbari Sari A, Olyaeemanesh A, Haghighi H, Aarabi M. COVID-19 pandemic and comparative health policy learning in iran. Arch Iran Med 2020;23:220-34.

7. Lai CC, Shih TP, Ko WC, Tang HJ, Hsueh PR. Severe acute respiratory syndrome coronavirus 2 (SARS-cov-2) and coronavirus disease-2019 (COVID-19): The epidemic and the challenges. Int J Antimicrob Agents 2020;55:105924.

8. Available from: https://www.worldometers.info/coronavirus/. [Last accessed on 2020 Apr 28].

9. Prompetchara E, Ketloy C, Palaga T. Immune responses in COVID-19 and potential vaccines: Lessons learned from SARS and MERS epidemic. Asian Pac J Allergy Immunol 2020;38:1-9.

10. Wang B, Li R, Lu Z, Huang Y. Does comorbidity increase the risk of patients with COVID-19: Evidence from meta-analysis. Aging (Albany NY) 2020;12:6049-57.

11. Marsh K, Eaton JW, Mahy M, Sabin K, Autenrieth CS, Wanyeki I, et al. Global, regional and country-level 90-90-90 estimates for 2018: Assessing progress towards the 2020 target. AIDS 2019;33:S213-26.

12. Wang $X, X u$ W, Hu G, et al. SARS-CoV-2 infects T lymphocytes through its spike protein-mediated membrane fusion. Cell Mol Immunol 2020:1-3.

13. Murad MH, Sultan S, Haffar S, Bazerbachi F. Methodological quality and synthesis of case series and case reports. BMJ Evid Based Med 2018;23:60-3.
14. Altuntas Aydin O, Kumbasar Karaosmanoglu H, Kart Yasar K. HIV/SARSCoV-2 co-infected patients in Istanbul, Turkey. J Med Virol 2020. [Ahead of print]

15. Wanga M, Luob L, Buc H, Xiad H. Case report: One case of coronavirus disease 2019(COVID-19) in patient co-infected by HIV with a low CD4+ T cell count. IJID 2020;96:148-50.

16. Riva A, Conti F, Bernacchia D, Pezzati L, Sollima S, Merli S, et al. Darunavir does not prevent SARS-cov-2 infection in HIV patients. Pharmacol Res 2020;157:104826.

17. Blanco JL, Ambrosioni J, Garcia F, Martínez E, Soriano A, Mallolas J, et al.; COVID-19 in HIV Investigators. COVID-19 in patients with HIV: Clinical case series. Lancet HIV 2020;7:e314-6.

18. Chen J, Cheng $X$, Wang R, Zeng $X$. Computed tomography imaging of an HIV-infected patient with coronavirus disease 2019 (COVID-19). J Med Virol 2020. [Ahead of print]

19. Zhao J, Liao X, Wang H, Wei L, Xing M, Liu L, Zhang Z. Early virus clearance and delayed antibody response in a case of COVID-19 with a history of co-infection with HIV-1 and HCV. Clin Infect Dis 2020. [Ahead of print]

20. Zhu F, Cao Y, Xu S, Zhou M. Co-infection of SARS-CoV-2 and HIV in a patient in Wuhan city, China. J Med Virol 2020;92:529-30.

21. Kolifarhood G, Aghaali M, Mozafar Saadati H, Taherpour N, Rahimi S, Izadi $\mathrm{N}$, et al. Epidemiological and clinical aspects of COVID-19; a narrative review. Arch Acad Emerg Med 2020;8:e41.

22. Lim J, Jeon S, Shin HY, Kim MJ, Seong YM, Lee WJ, et al. Case of the index patient who caused tertiary transmission of COVID-19 infection in Korea: The application of lopinavir/ritonavir for the treatment of COVID-19 infected pneumonia monitored by quantitative RT-PCR. J Korean Med Sci 2020;35:e79.

23. Cao B, Wang Y, Wen D, Liu W, Wang J, Fan G, et al. A trial of lopinavirritonavir in adults hospitalized with severe covid-19. N Engl J Med 2020;382:1787-99.

24. Center for Disease Control and Prevention (CDC): What to know about COVID-19 and HIV. Accessed on May 2, 2020 [https://www.cdc.gov/ coronavirus/2019-ncov/need-extra-precautions/hiv.html].

25. He R, Lu Z, Zhang L, Fan T, Xiong R, Shen X, et al. The clinical course and its correlated immune status in COVID-19 pneumonia. J Clin Virol 2020;127:104361.

26. Mascolo S, Romanelli A, Carleo MA, Esposito V. Could HIV infection alter the clinical course of SARS-CoV-2 infection? When less is better. J Med Virol 2020. [Ahead of print]

27. Tahamtan A, Ardebili A. Real-time RT-PCR in COVID-19 detection: Issues affecting the results. Expert Rev Mol Diagn 2020;20:453-4.

28. Ivashkiv LB, Donlin LT. Regulation of type I interferon responses. Nat Rev Immunol 2014;14:36-49.

29. Lee HK, Lee BH, Seok SH, Baek MW, Lee HY, Kim DJ, et al. Production of specific antibodies against SARS-coronavirus nucleocapsid protein without cross reactivity with human coronaviruses $229 \mathrm{E}$ and OC43. J Vet Sci 2010;11:165-7.

30. Li Z, Yi Y, Luo X, Xiong N, Liu Y, Li S, et al. Development and clinical application of a rapid IgM-IgG combined antibody test for SARS-CoV-2 infection diagnosis. J Med Virol 2020. [Ahead of print]

31. Bao C, Liu X, Zhang H, Li Y, Liu J. Coronavirus disease 2019 (COVID-19) CT findings: A systemic review and meta-analysis. J Am Coll Radiol 2020;17:701-9. 